NBI-HE-93-18

UTHEP-255

July, 1993

\title{
EXTENDED SUPERCONFORMAL ALGEBRAS AND FREE FIELD REALIZATIONS FROM HAMILTONIAN REDUCTION
}

\author{
KATSUSHI ITO \\ Institute of Physics, University of Tsukuba \\ Ibaraki 305, Japan \\ JENS OLE MADSEN \\ and \\ JENS LYNG PETERSEN \\ Niels Bohr Institute, University of Copenhagen, \\ Blegdamsvej 17, DK 2100 Copenhagen Ø, Denmark
}

\begin{abstract}
We develop the method of the hamiltonian reduction of affine Lie superalgebras to obtain explicit and general expressions both for the classical and the quantum extended superconformal algebras. By performing the gauge transformation which connects the diagonal gauge with the Drinfeld-Sokolov gauge and considering the quantum corrections, we get generic expressions for the classical and quantum free field realizations of the algebras.
\end{abstract}


Recently, there has been a renewal of interest in the non-linearly extended superconformal algebras, originally introduced by Knizhnik and Bershadsky [1]. Two of the present authors have considered the classification of extended superconformal algebras in the context of hamiltonian reduction ([2, 3, 4, 5]) of Lie superalgebras in [6], and the present authors have further explored this, both in the special case of the so called doubly extended $N=4$ algebra $\tilde{A}_{\gamma}[7]$ and in the general case [8, 9]. These algebras have also been classified independently by Bowcock and by Fradkin and Linetsky [10] from a purely algebraic approach.

The method of hamiltonian reduction clarifies the integrable structures of the hierarchy of the relevant soliton equations [2]. From the point of view of conformal field theories, this approach provides a powerful technique to obtain the free field realizations of the algebras. These free field realizations are useful for studying the representation of the algebra and correlation functions.

In the present paper we develop the technique of hamiltonian reduction to present simple and completely general expressions for the algebras. We find the explicit gauge transformation which gives the classical free field realization. We then obtain a quantum expression from the requirement of the closure of the algebras. The difference with the classical expression may be expressed as a "renormalization" of certain constants. Some preliminary results have been reported in ref. [7].

Let $\mathbf{g}=\mathbf{g}_{0} \oplus \mathbf{g}_{1}$ be a rank $n$ basic classical Lie superalgebra with an even subalgebra $\mathbf{g}_{0}$ and an odd subspace $\mathbf{g}_{1}$. As described in [6, 8, 9], we consider the case $\mathbf{g}_{0}=G \oplus A_{1}$, in which we use only the $A_{1}$ subalgebra for the reduction. The generators of the Lie superalgebra split into $A_{1}$ representations with spins $\left\{s_{i}\right\}$, and the conformal dimensions for a current in the reduced algebra are $\left\{1+s_{i}\right\}$. The fact that $\mathbf{g}_{0}=G \oplus A_{1}$ implies that the bosonic sector of the algebra contains an energy-momentum tensor of conformal dimension 2 and some dimension 1 affine $\widehat{G}$ currents. In the superalgebras listed in table 1, the odd subspace carries spin $\frac{1}{2}$ representations of the $A_{1}$, and therefore gives rise to fermionic supercurrents of conformal dimension $\frac{3}{2}$ after the reduction. In the case of the Lie superalgebra $B(1 \mid n)$, where the even subalgebra is of the form $\mathbf{g}_{0}=C_{n} \oplus A_{1}$, the odd subspace carries spin 1 representations of the $A_{1}$, giving rise to fermionic currents with integer spin in the reduced algebra. This type of algebra falls outside of the scope of this paper, and we will therefore not consider $B(1 \mid n)$ here.

We introduce some notation. We denote by $\Delta=\Delta^{0} \cup \Delta^{1}$ the set of roots of g, where $\Delta^{0}\left(\Delta^{1}\right)$ is the set of even (odd) roots. (The root systems of those Lie superalgebras that we are interested were given for example in [8]). Denote the set of positive even (odd) roots by $\Delta_{+}^{0}\left(\Delta_{+}^{1}\right)$. The superalgebra $\mathrm{g}$ has a canonical basis $\left\{E_{\alpha}, e_{\gamma}, h^{i}\right\}\left(\alpha \in \Delta^{0}, \gamma \in \Delta^{1}, i=1, \ldots, n\right)$, which satisfies (anti-)commutation relations

$$
\left[E_{\alpha}, E_{\beta}\right]= \begin{cases}N_{\alpha, \beta} E_{\alpha+\beta}, & \text { for } \alpha, \beta, \alpha+\beta \in \Delta^{0} \\ \frac{2 \alpha \cdot h}{\alpha^{2}}, & \text { for } \alpha+\beta=0\end{cases}
$$




$$
\begin{aligned}
\left\{e_{\gamma}, e_{\gamma^{\prime}}\right\} & =N_{\gamma, \gamma^{\prime}} E_{\gamma+\gamma^{\prime}}, \quad \text { for } \gamma, \gamma^{\prime} \in \Delta^{1}, \gamma+\gamma^{\prime} \in \Delta^{0} \\
\left\{e_{\gamma}, e_{-\gamma}\right\} & =\gamma \cdot h, \quad \text { for } \gamma \in \Delta_{+}^{1} \\
{\left[e_{\gamma}, E_{\alpha}\right] } & =N_{\gamma, \alpha} e_{\gamma+\alpha}, \quad \text { for } \alpha \in \Delta^{0}, \gamma, \gamma+\alpha \in \Delta^{1} \\
{\left[h^{i}, E_{\alpha}\right] } & =\alpha^{i} E_{\alpha}, \quad\left[h^{i}, e_{\gamma}\right]=\gamma^{i} e_{\gamma} .
\end{aligned}
$$

The even subalgebra $\mathbf{g}_{0}$ is generated by $\left\{E_{\alpha}, h^{i}\right\}$. The odd subspace $\mathbf{g}_{1}$ is spanned by $\left\{e_{\gamma}\right\} . \mathbf{g}_{0}$ acts on $\mathbf{g}_{1}$ as a faithful, completely reducible representation. The Killing form $($, ) on $\mathbf{g}$ is defined by

$$
\left(E_{\alpha}, E_{\beta}\right)=\frac{2}{\alpha^{2}} \delta_{\alpha+\beta, 0}, \quad\left(e_{\gamma}, e_{-\gamma^{\prime}}\right)=-\left(e_{-\gamma}, e_{\gamma^{\prime}}\right)=\delta_{\gamma, \gamma^{\prime}}, \quad\left(h^{i}, h^{j}\right)=\delta_{i j},
$$

for $\alpha, \beta \in \Delta^{0}, \gamma, \gamma^{\prime} \in \Delta_{+}^{1}, i, j=1, \ldots, n$.

In terms of these generators, we can write the expression for the affine current as

$$
J(z)=\sum_{\alpha \in \Delta^{0}} \frac{\alpha^{2}}{2} J_{\alpha}(z) E_{\alpha}+\sum_{\gamma \in \Delta^{1}} j_{\gamma}(z) e_{\gamma}+\sum_{i=1}^{n} H^{i}(z) h^{i} .
$$

On the space of currents, the Poisson bracket structure is introduced by the infinitesimal gauge transformations $\delta J(z)=[\Lambda(z), J(z)]+k \partial \Lambda(z)$, where $\Lambda(z) \in \mathbf{g}$ and $k$ is the level of the affine Lie superalgebra $\hat{\mathrm{g}}$.

In the present case, the odd subspace $\mathbf{g}_{1}$ belongs to the spin $\frac{1}{2}$ representation with respect to the even subalgebra $A_{1}$ and splits into two parts $\mathbf{g}_{1}=\left(\mathbf{g}_{1}\right)_{+\frac{1}{2}} \oplus\left(\mathbf{g}_{1}\right)_{-\frac{1}{2}}$. By choosing appropriate simple roots for $\mathbf{g}$, we can take the root space $\Delta_{+\frac{1}{2}}^{1}$, which spans $\left(\mathbf{g}_{1}\right)_{+\frac{1}{2}}$, to be the space of odd positive roots $\Delta_{+}^{1}=\Delta_{+\frac{1}{2}}^{1}$, and we find the relation $\Delta_{+}^{1}=\Delta_{-}^{1}+\theta$.

Denote the set of roots of the subalgebra $G$ by $\Delta(G)$. Each odd space $\left(\mathbf{g}_{1}\right)_{ \pm \frac{1}{2}}$ belongs to a fundamental representation of $G$ of dimension $\left|\Delta_{+}^{1}\right|$, this being the number of positive odd roots. If we denote the representation matrices in the canonical basis by $t_{\gamma, \gamma^{\prime}}^{\alpha}, t_{\gamma, \gamma^{\prime}}^{i}$ then we have (using the Jacobi identities) for $\gamma \in \Delta_{+}^{1}[9]$

$$
\begin{aligned}
t_{\gamma, \gamma^{\prime}}^{\alpha} & =-N_{\alpha,-\gamma} \delta_{\gamma-\alpha, \gamma^{\prime}} \\
t_{\gamma, \gamma^{\prime}}^{i} & =\left(\gamma_{\perp}\right)^{i} \delta_{\gamma, \gamma^{\prime}}, \\
\operatorname{tr}\left(t^{i} t^{j}\right) & =c_{F} \delta_{i j} \\
\operatorname{tr}\left(t^{\alpha} t^{\beta}\right) & =c_{F} \frac{2}{\alpha^{2}} \delta_{\alpha+\beta, 0}, \\
t_{\gamma, \gamma^{\prime}}^{\alpha} & =-t_{\theta-\gamma^{\prime}, \theta-\gamma}^{\alpha}, \\
t_{\gamma, \gamma^{\prime}}^{\alpha} & =t_{\gamma^{\prime}, \gamma}^{-\alpha} .
\end{aligned}
$$

where $\gamma_{\perp}$ is the projection of $\gamma \in \Delta_{+}^{1}$ on the root space of $G$ and the constant $c_{F}$ is defined as $\sum_{\gamma \in \Delta_{+}^{1}} \gamma_{\perp}^{2} / \operatorname{rank}(G)$. It is convenient to introduce the affine $\widehat{G}$ currents in 
the above basis (4):

$$
J_{\gamma, \gamma^{\prime}}(z) \equiv \sum_{\alpha \in \Delta(G)} \frac{\alpha^{2}}{2} t_{\gamma, \gamma^{\prime}}^{\alpha} J_{-\alpha}(z)+\sum_{i=1}^{\operatorname{rank}(G)} t_{\gamma, \gamma^{\prime}}^{i} H_{i}(z)
$$

The procedure of hamiltonian reduction is to impose the condition $J_{-\theta}(z)=1$ on the current, where $-\theta$ is the negative root in the $A_{1}$ subalgebra. With this constraint, there is still a gauge invariance, which allows one to choose one or the other gauge slice as a representative of the reduced phase space, for example the so-called "DrinfeldSokolov (DS)" gauge or the diagonal gauge. In the DS gauge the current is

$$
J_{D S}(z)=\frac{\theta^{2}}{2} E_{-\theta}+\frac{\theta^{2}}{2} T(z) E_{\theta}+\sum_{\gamma \in \Delta_{+}^{1}} G_{\gamma}(z) e_{\gamma}+\sum_{\alpha \in \Delta(G)} \frac{\alpha^{2}}{2} J_{\alpha}(z) E_{\alpha}+\sum_{i=1}^{\operatorname{rank}(G)} H^{i} h^{i} .
$$

The generic gauge transformation projected on the DS gauge slice becomes the transformation corresponding to the extended superconformal algebra, see [6, 8] for details. the Poisson bracket structure on the reduced phase space can be conveniently written in terms of formal "operator product expansions". We define the total energymomentum tensor to be $T_{E S A}=\frac{\theta^{2}}{2 k} T+T_{\text {Sugawara }}$, where $T$ is the generator occurring in the DS current, and

$$
\begin{aligned}
T_{\text {Sugawara }} & =\frac{1}{2 k}\left\{\sum_{\alpha \in \Delta(G)} \frac{\alpha^{2}}{2} J_{\alpha} J_{-\alpha}+\sum_{i=1}^{\operatorname{rank}(G)} H^{i} H^{i}\right\} \\
& =\frac{1}{2 k c_{F}} \operatorname{tr}\left(J^{2}\right),
\end{aligned}
$$

and we rescale the supercurrents as $\tilde{G}_{\gamma}=\mathrm{i} \sqrt{\frac{\theta^{2}}{2 k}} G_{\gamma}$. The result is the classical extended superconformal algebra:

$$
\begin{aligned}
T_{E S A}(z) T_{E S A}(w) & =\frac{\frac{-6 k}{\theta^{2}}}{(z-w)^{4}}+\frac{2 T_{E S A}(w)}{(z-w)^{2}}+\frac{\partial T_{E S A}(w)}{z-w}+\cdots \\
T_{E S A}(z) \tilde{G}_{\gamma}(w) & =\frac{\frac{3}{2} \tilde{G}_{\gamma}(w)}{(z-w)^{2}}+\frac{\partial \tilde{G}_{\gamma}(w)}{z-w}+\cdots, \\
J_{\beta}(z) \tilde{G}_{\gamma}(w) & =\frac{t_{\gamma, \gamma^{\prime}}^{\beta} \tilde{G}_{\gamma^{\prime}}(w)}{z-w}+\cdots, \quad H^{i}(z) \tilde{G}_{\gamma}(w)=\frac{t_{\gamma, \gamma^{\prime}}^{i} \tilde{G}_{\gamma^{\prime}}(w)}{z-w}+\cdots \\
\tilde{G}_{\theta-\gamma^{\prime}}(z) \tilde{G}_{\gamma}(w) & =-\frac{2 k \delta_{\gamma^{\prime}, \gamma}}{(z-w)^{3}}+\left(2 \frac{J_{\gamma, \gamma^{\prime}}(w)}{(z-w)^{2}}+\frac{\partial J_{\gamma, \gamma^{\prime}}(w)}{z-w}\right) \\
& +\frac{\theta^{2}}{2} \delta_{\gamma, \gamma^{\prime}} \frac{T_{E S A}(w)-T_{\text {Sugawara }}(w)}{z-w}-\frac{1}{k} \frac{\left(J^{2}\right)_{\gamma, \gamma^{\prime}}(w)}{z-w}+\cdots
\end{aligned}
$$

Here we imply the notation:

$$
\left(J^{2}\right)_{\gamma, \gamma^{\prime}} \equiv \sum_{\gamma^{\prime \prime} \in \Delta_{+}^{1}} J_{\gamma, \gamma^{\prime \prime}} J_{\gamma^{\prime \prime}, \gamma}
$$


Notice that this expression is nonvanishing only if either $\gamma-\gamma^{\prime} \in \Delta(G), \gamma=\gamma^{\prime}$ or $\gamma+\gamma^{\prime}=\theta$. We note that the classical value of the central charge $c_{E S A}$ is $-12 k / \theta^{2}$. The operator product expansions of the affine $\widehat{G}$ (or $\widehat{G}=\oplus_{i} \widehat{G}^{i}$ ) currents are the standard ones at level $K^{(i)}=\frac{2 k}{\alpha_{L i}^{2}}$, where $\alpha_{L}$ denotes the "long root".

There are several methods which allow us to obtain the classical free field realization of the extended Virasoro algebras. One method is based on the Miura transformation, used in the case of hamiltonian reduction for non-exceptional simple Lie algebras [2]. This formulation has been generalized to the extended superconformal algebras for Lie superalgebras $s l(N \mid 2)$ and $\operatorname{ssp}(N \mid 2)$ [6]. However for the remaining Lie (super)algebras, especially exceptional type algebras, it is difficult to find the explicit form of the Miura transformations. Another method consists in finding the explicit gauge transformation from the diagonal gauge, in which the Poisson structure of the free fields are defined, to the Drinfeld-Sokolov gauge. We show that this last method provides a general procedure for giving the free field realization for arbitrary extended algebras.

Let us consider the gauge transformation $J \rightarrow J^{g}=g J g^{-1}+k \partial g g^{-1}$. By parametrizing a supergroup element $g$ by $e^{\Lambda}(\Lambda \in \mathbf{g})$, we get

$$
J^{g}=\sum_{n=0}^{\infty} \frac{1}{n !}\left\{(\operatorname{ad} \Lambda)^{n} J+k(\operatorname{ad} \Lambda)^{n-1} \partial \Lambda\right\} .
$$

Here $(\operatorname{ad} X) Y=[X, Y]$ for $X, Y \in \mathbf{g}$. We write the current in the diagonal gauge as $J_{\operatorname{diag}}(z)=\sum_{\alpha \in \Delta(G)} \frac{\alpha^{2}}{2} \hat{J}_{\alpha}(z) E_{\alpha}+\sum_{i} \hat{H}_{i}(z) h^{i}+\sum_{\gamma \in \Delta_{+}^{1}} \mathrm{i} \sqrt{\frac{\theta^{2}}{2}} \chi_{\theta-\gamma}(z) e_{-\gamma}-\mathrm{i} \sqrt{k} \partial \phi(z) h_{\theta}+\frac{\theta^{2}}{2} E_{-\theta}$,

where $\chi_{\gamma}$ are complex fermions, normalized to

$$
\chi_{\gamma}(z) \chi_{\theta-\gamma^{\prime}}(w)=\frac{\delta_{\gamma, \gamma^{\prime}}}{z-w}+\cdots
$$

and $\phi$ is a free boson coupled to a background charge $q=-2 \sqrt{\frac{k}{\theta^{2}}}$ with $\phi(z) \phi(w)=$ $-\ln (z-w)+\cdots$. The energy-momentum tensor corresponding to $\phi$ is $T_{\phi}=-\frac{1}{2}(\partial \phi)^{2}-$ $\frac{i q}{2} \partial^{2} \phi$. The currents $\hat{J}$ are affine $\hat{G}$ currents that commute with the fermions. The level of the $\hat{J}$ 's are $K=\frac{2}{\alpha_{L}^{2}} k$. The $\hat{J}$ 's can be built based on the standard Wakimoto construction [11, 12], but the explicit free field realization of the currents is not necessary in the present paper. Using various equations for the structure constants of the Lie superalgebra [8], it is straightforward to calculate $\left(J_{\text {diag }}\right)^{g}$ with $g=e^{\Lambda}$ and

$$
\Lambda(z)=\sum_{\gamma \in \Delta_{+}^{1}} \xi_{\gamma}(z) e_{\gamma}+\varepsilon(z) E_{\theta}
$$

which generates the residual gauge symmetry. The gauge transformation (10) truncates at fourth order for $J=J_{\text {diag }}$. The truncation at finite order is a general feature 
of the technique of hamiltonian reduction. The condition

$$
J_{D S}=\left(J_{\text {diag }}\right)^{g}
$$

determines $\Lambda$ to be $\Lambda=-\mathrm{i} \sqrt{\frac{2}{\theta^{2}}} \sum_{\gamma} \chi_{\theta-\gamma} e_{\gamma}+\mathrm{i} \sqrt{\frac{k}{\theta^{2}}} \partial \phi E_{\theta}$ and we find

$$
\begin{aligned}
J_{D S}= & \mathrm{i} \sqrt{\frac{2}{\theta^{2}}} \sum_{\gamma \in \Delta_{+}^{1}}\left\{-k \partial \chi_{\gamma}-\frac{\mathrm{i}}{\sqrt{2}} \sqrt{\frac{k \theta^{2}}{2}} \partial \phi \chi_{\gamma}-\hat{J}_{\gamma, \gamma^{\prime}} \chi_{\gamma^{\prime}}-\frac{2}{3} J_{\gamma, \gamma^{\prime}}^{f} \chi_{\gamma^{\prime}}\right\} e_{\theta-\gamma} \\
& +\sum_{\alpha} \frac{\alpha^{2}}{2}\left(\hat{J}_{\alpha}+J_{\alpha}^{f}\right) E_{\alpha}+\sum_{i}\left(\hat{H}_{i}+H_{i}^{f}\right) h^{i} \\
& +k\left(-\frac{1}{2}(\partial \phi)^{2}+\mathrm{i} \sqrt{\frac{k}{\theta^{2}}} \partial^{2} \phi-\frac{1}{2 k c_{F}} \operatorname{tr}\left(J^{f}\right)^{2}-\frac{1}{k c_{F}} \operatorname{tr}\left(\hat{J} J^{f}\right)+\sum_{\gamma \in \Delta_{+}^{1}} \partial \chi_{\theta-\gamma} \chi_{\gamma}\right) E_{\theta} .
\end{aligned}
$$

Comparing (6) and (14) we get the free field representation of the classical extended superconformal algebras. The energy momentum tensor and the supercurrents are given by

$$
\begin{aligned}
T_{E S A} & =-\frac{1}{2}(\partial \phi)^{2}+\mathrm{i} \sqrt{\frac{k}{\theta^{2}}} \partial^{2} \phi+\hat{T}_{\text {Sugawara }}+\frac{1}{2} \sum_{\gamma \in \Delta_{+}^{1}} \partial \chi_{\theta-\gamma} \chi_{\gamma} \\
\tilde{G}_{\gamma} & =+\sqrt{k} \partial \chi_{\gamma}+\frac{\mathrm{i}}{\sqrt{2}} \sqrt{\frac{\theta^{2}}{2}} \partial \phi \chi_{\gamma}-\frac{1}{\sqrt{k}} \sum_{\gamma^{\prime} \in \Delta_{+}^{1}}\left(\hat{J}_{\gamma, \gamma^{\prime}}+\frac{2}{3} J_{\gamma, \gamma^{\prime}}^{f}\right) \chi_{\gamma^{\prime}}
\end{aligned}
$$

The free field realizations of the affine currents $J_{\alpha}$ and $H^{i}$ are just the coefficients of $\frac{\alpha^{2}}{2} E_{\alpha}$ and $h^{i}$ respectively :

$$
\begin{aligned}
J_{\alpha} & =\hat{J}_{\alpha}+J_{\alpha}^{f}, \\
H^{i} & =\hat{H}_{i}+H_{i}^{f},
\end{aligned}
$$

where $J_{\alpha}^{f}$ and $H_{i}^{f}$ are Kac-Moody currents constructed from the fermions in the standard way

$$
\begin{aligned}
J_{\alpha}^{f} & =\frac{1}{2} \sum_{\gamma^{\prime}, \gamma \in \Delta_{+}^{1}} t_{\gamma^{\prime}, \gamma}^{\alpha} \chi_{\gamma} \chi_{\theta-\gamma^{\prime}} \\
H_{i}^{f} & =\frac{1}{2} \sum_{\gamma^{\prime}, \gamma \in \Delta_{+}^{1}} t_{\gamma^{\prime}, \gamma}^{i} \chi_{\gamma} \chi_{\theta-\gamma^{\prime}}
\end{aligned}
$$

We now use the classical free field realizations to write down an ansatz for the generators of the quantum algebra algebra, in which we allow for arbitrary coefficients on the various terms. We then calculate the quantum operator product expansions of these generators. This way we find the coefficients and the modifications in the 
algebra and the free field realizations necessary for the closure of the quantum algebra. Define $\chi_{\gamma}$ and $\hat{J}$ as above, and define $\phi$ to be coupled to the background charge $Q=-\frac{2}{\sqrt{\theta^{2}} \alpha_{+}}\left(k+\frac{\theta^{2}}{2}\right)$, where $\alpha_{+}=\sqrt{k+h^{\vee}} . h^{\vee}$ is the dual Coxeter number of the Lie superalgebra $\mathbf{g}$. The affine currents $J_{\alpha}$ and $H^{i}$ with level $K=\frac{2}{\alpha_{L}^{2}}\left(k-\frac{c_{F}}{2}\right)$ are constructed as in eq. (16) ¿from the affine currents $\hat{J}_{\alpha}$ and $\hat{H}^{i}$ from the free field representation, with level $\hat{K}=\frac{2}{\alpha_{L}^{2}}\left(k-c_{F}\right)$, and the affine currents $J_{\alpha}^{f}$ and $H_{f}^{i}$ constructed from the fermions, with level $K^{f}=\frac{c_{F}}{\alpha_{L}^{2}}$. For the explicit realizations of the affine currents $\hat{J}_{\alpha}$ and $\hat{H}^{i}$ in terms of free fields we refer to ref. [11] or [12]. The quantum free field realizations of the supercurrents are found to be

$G_{\gamma}(z)=\frac{\left(k+\theta^{2} / 2\right)}{\alpha_{+}} \partial \chi_{\gamma}+\frac{i}{\sqrt{2}} \sqrt{\frac{\theta^{2}}{2}} \partial \phi(z) \chi_{\gamma}-\frac{1}{\alpha_{+}} \sum_{\gamma^{\prime} \in \Delta_{+}^{1}}\left(\hat{J}_{\gamma, \gamma^{\prime}}(z) \chi_{\gamma^{\prime}}(z)+\frac{2}{3}: J_{\gamma, \gamma^{\prime}}^{f} \chi_{\gamma^{\prime}}:(z)\right)$

The quantum energy-momentum tensor is $T_{E S A}(z)=T_{\phi}(z)+T_{\chi}(z)+\hat{T}_{\text {Sugawara }}(z)$, where

$$
\begin{aligned}
T_{\phi}(z) & =-\frac{1}{2}:(\partial \phi)^{2}:(z)-\frac{i Q}{2} \partial^{2} \phi(z), \\
\hat{T}_{\text {Sugawara }}(z) & =\frac{1}{2 \alpha_{+}^{2}}\left\{\sum_{\alpha \in \Delta(G)} \frac{\alpha^{2}}{2}: \hat{J}_{\alpha} \hat{J}_{-\alpha}:(z)+\sum_{i=1}^{\operatorname{rank}(G)}: \hat{H}^{i} \hat{H}^{i}:(z)\right\}, \\
T_{\chi}(z) & =\frac{1}{2} \sum_{\gamma \in \Delta_{+}^{1}}: \partial \chi_{\theta-\gamma} \chi_{\gamma}:(z) .
\end{aligned}
$$

The quantum algebra is very similar to the classical one. The central charge of the quantum algebra is shifted from $-\frac{12 k}{\theta^{2}}$ to $-\frac{12 k}{\theta^{2}}+\frac{k \text { sdimg }}{k+h^{\vee}}-2-\frac{1}{2}\left|\Delta_{+}^{1}\right|$ where sdimg is the superdimension of $\mathbf{g}$, and as already mentioned above, the level of the affine currents is shifted from $K_{c l}=\frac{2}{\alpha_{L}^{2}} k$ to $K=\frac{2}{\alpha_{L}^{2}}\left(k-\frac{c_{F}}{2}\right)$. The OPE between the supercurrents are modified into

$$
\begin{aligned}
G_{\theta-\gamma^{\prime}}(z) G_{\gamma}(w) & =\frac{-f(k)}{k+h^{\vee}} \frac{\delta_{\gamma^{\prime}, \gamma}}{(z-w)^{3}}+\frac{1}{k+h^{\vee}} \sum_{i} g^{(i)}(k)\left(2 \frac{J_{\gamma, \gamma^{\prime}}^{(i)}(w)}{(z-w)^{2}}+\frac{\partial J_{\gamma, \gamma^{\prime}}^{(i)}(w)}{z-w}\right) \\
& +\frac{\theta^{2}}{2} \delta_{\gamma, \gamma^{\prime}} \frac{T_{E S A}(w)-T_{G}(w)}{z-w}-\frac{1}{k+h^{\vee}} \sum_{i} \frac{:\left(J^{2}\right)_{\gamma, \gamma^{\prime}:_{S}}(w)}{z-w}+\cdots .(20)
\end{aligned}
$$

Here $J_{\gamma, \gamma^{\prime}}^{(i)}$ denotes the part of the current $J_{\gamma, \gamma^{\prime}}$ associated with the affine algebra $\widehat{G}^{(i)}$. The functions $f(k)$ and $g(k)$ are given by

$$
\begin{aligned}
f(k) & =2\left(k+\frac{\theta^{2}}{2}+\frac{\alpha_{L}^{2}}{4} H^{\vee}\right)\left(k-\frac{c_{F}}{2}\right), \\
g^{(i)}(k) & =2\left(k+\frac{\theta^{2}}{2}+\frac{\left(\alpha_{L}^{(i)}\right)^{2}}{4}\left(H^{\vee}\right)^{(i)}\right),
\end{aligned}
$$


In this expression it is not obvious that $f(k)$ is well defined in the case where $G=$ $G^{(1)} \oplus G^{(2)}$. However, one finds the identity

$$
\frac{\theta^{2}}{2}+\left(\frac{\alpha_{L}^{2}}{4} H^{\vee}\right)^{(1)}=-\frac{c_{F}^{(2)}}{2}
$$

and similarly when (1) and (2) is interchanged. One easily checks that this does indeed imply that $f(k)$ is well defined.

We note that in the classical limit $k \rightarrow \infty$ we recover the classical expression (8).

$$
T_{G}(w) \equiv \frac{1}{2\left(k+h^{\vee}\right)}\left\{\sum_{\alpha \in \Delta(G)} \frac{\alpha^{2}}{2}: J_{\alpha} J_{-\alpha}:(w)+\sum_{i}: H_{i} H_{i}:(w)\right\}
$$

is similar to the Sugawara energy momentum tensor, but in fact carries a different normalization, namely the normalization that would be needed for the "hat" part whereas here we are dealing with the full generators. In order to present the algebra in the above way which is related so simply to the classical expressions, it is important to use the symmetrized normal ordering prescription in the quadratic terms in the expression for the ope $G_{\theta-\gamma^{\prime}}(z) G_{\gamma}(w)$ :

$$
: A(z) B(z):_{S} \equiv \frac{1}{2} \oint_{z} \frac{d w}{2 \pi i} \frac{A(z) B(w)+A(w) B(z)}{z-w} .
$$

In this paper we have employed a unified formalism for treating those superconformal algebras which are obtainable by hamiltonian reduction of Lie superalgebras, using the techniques developed in refs. [6, 7, 8]. The new results of the present paper are that we have obtained expressions for the algebras and for the free field realizations of these algebras, which are not only completely general, but also very simple compared to previous expressions. We have provided formulas both for the classical (8, 15) and the quantum $(18,19,20)$ cases. The two are quite similar but differ as expected by certain "renormalizations" of constants for which we have obtained the general form depending on certain group theory parameters. As a particular new result in this paper we have obtained the classical expressions for the free field realizations in a very straight forward way, simply by providing an explicit expression for the gauge transformation connecting the diagonal gauge (11) and the Drinfeld-Sokolov gauge (14). This method would seem to be useful for finding the free field realization for other extended algebras.

The study of conformal field theories with extended chiral algebras containing a superconformal algebra, is expected to be of interest from various points of view. Thus the corresponding target manifold in a string theory application would be expected to have a rich geometrical structure. For actual applications we need more information on the representation theory of these algebras. Some crucial steps towards an understanding of the representations were taken in [7, 8] where we considered the structure of the screening operators and the null vectors of these algebras. But more work in 
Table 1: Constants occurring in the free field realizations.

\begin{tabular}{|l|lrrrrrr|}
\hline \hline $\mathrm{g}$ & $G$ & $\theta^{2} / 2$ & $h^{\vee}$ & $\left(\frac{\alpha_{L}^{2}}{2} H^{\vee}\right)^{(1)}$ & $\left(\frac{\alpha_{L}^{2}}{2} H^{\vee}\right)^{(2)}$ & $c_{F}^{(1)}$ & $c_{F}^{(2)}$ \\
\hline$B(n \mid 1)$ & $B_{n}$ & 2 & $-2 n+3$ & $-2 n+1$ & & -2 & \\
$D(n \mid 1)$ & $D_{n}$ & 2 & $-2 n+4$ & $-2 n+2$ & -2 & \\
$F(4)$ & $B_{3}$ & $\frac{2}{3}$ & -3 & -5 & & -2 & \\
$G(3)$ & $G_{2}$ & $-\frac{4}{3}$ & 2 & 4 & & 2 & \\
$A(n \mid 1)$ & $A_{n} \oplus u(1)$ & -1 & $n-1$ & $n+1$ & 0 & 2 & $-n+1$ \\
$D(2 \mid n)$ & $C_{n} \oplus A_{1}$ & -1 & $2 n-2$ & $2 n+2$ & -2 & 4 & $-2 n$ \\
$D(2 \mid 1 ; \alpha)$ & $A_{1} \oplus A_{1}$ & 1 & 0 & $-2 \gamma$ & $-2(1-\gamma)$ & $-2 \gamma$ & $-2(1-\gamma)$ \\
\hline
\end{tabular}

this direction remains. Furthermore it can be expected that for actual applications the relevant chiral algebra will contain one of the superconformal algebras described here (i.e. with generators of dimensions $1,3 / 2$ and 2, only) as a subalgebra. This points to the much larger study of super- $W$-algebras and their classification. A particularly interesting relation should exist between the algebras we have studied here and the ones considered in [13] where the extended superconformal algebras obtainable from a similar but supersymmetric hamiltonian reduction have been classified. Indeed the two classifications agree in many ways. However that formalism results in algebras with generators appearing as supersymmetry multiplets. The relation between that formalism, and the one we use in this paper has recently been considered in [14].

Acknowledgements: This work was supported in part by EEC contract no. SC1 0394 C (EDB). One of the authors (K.I.) would like to thank the Niels Bohr Institute for its financial support during his stay. The free field realizations in this paper has been checked using the Mathematica operator product expansion package constructed by Kris Thielemans. 


\section{References}

1. V.G. Knizhnik, Theor. Math. Phys. 66 (1986) 68; M. Bershadsky, Phys. Lett. B174 (1986) 285.

2. V.G. Drinfeld and V.V. Sokolov, J. Sov. Math. 30 (1984) 1975.

3. A.A. Belavin, in "Quantum String Theory" Proceedings in Physics vol. 31 (Springer Verlag, Berlin ,1989); A. Alekseev and S. Shatashvili, Nucl. Phys. B323 (1989) 719.

4. M. Bershadsky and H. Ooguri, Comm. Math. Phys. 126 (1989) 429.

5. J. Balog, L. Fehér, P. Forgács, L. O’Raifeartaigh and A. Wipf, Ann. Phys. 203 (1990) 76 .

6. K. Ito and J.O. Madsen, Phys. Lett. B283 (1992) 223.

7. K. Ito, J.O. Madsen and J.L. Petersen, Phys. Letters B292 (1992) 298.

8. K. Ito, J.O. Madsen and J.L. Petersen, Nucl. Phys. B398 (1993) 425.

9. K. Ito, J.O. Madsen and J.L. Petersen, NBI preprint NBI-HE-92-81; to appear in the proceedings of the International Workshop on "String Theory, Quantum Gravity and the Unification of the Fundamental Interactions", Rome, September 21-26 1992 .

10. P. Bowcock, Nucl. Phys. B381 (1992) 415. E.S. Fradkin and V.Ya. Linetskii, preprint ITP-SB-92-34, preprint ETH-TH/92-6.

11. M. Wakimoto, Comm. Math. Phys.104 (1986) 605; D. Bernard and G. Felder, Comm. Math. Phys.127 (1990) 145; B. Feigin and E. Frenkel, in "Physics and Mathematics of Strings", eds. L. Brink et al., (World Scientific, 1990 Singapore).

12. K. Ito and S. Komata, Mod. Phys. Lett. A 6 (1991) 581.

13. L. Frappat, E. Ragoucy and P. Sorba, preprint ENSLAPP-AL-391/92;

14. E. Ragoucy, preprint NORDITA-93-39-P; 